\title{
Efficient Cache Placement in Multi-Hop Wireless Networks
}

\author{
Pavan Nuggehalli, Member, IEEE, Vikram Srinivasan, Member, IEEE, Carla-Fabiana Chiasserini, Member, IEEE, \\ and Ramesh R. Rao, Senior Member, IEEE
}

\begin{abstract}
In this paper, we address the problem of efficient cache placement in multi-hop wireless networks. We consider a network comprising a server with an interface to the wired network, and other nodes requiring access to the information stored at the server. In order to reduce access latency in such a communication environment, an effective strategy is caching the server information at some of the nodes distributed across the network. Caching, however, can imply a considerable overhead cost; for instance, disseminating information incurs additional energy as well as bandwidth burden. Since wireless systems are plagued by scarcity of available energy and bandwidth, we need to design caching strategies that optimally trade-off between overhead cost and access latency. We pose our problem as an integer linear program. We show that this problem is the same as a special case of the connected facility location problem, which is known to be NP-hard. We devise a polynomial time algorithm which provides a suboptimal solution. The proposed algorithm applies to any arbitrary network topology and can be implemented in a distributed and asynchronous manner. In the case of a tree topology, our algorithm gives the optimal solution. In the case of an arbitrary topology, it finds a feasible solution with an objective function value within a factor of 6 of the optimal value. This performance is very close to the best approximate solution known today, which is obtained in a centralized manner. We compare the performance of our algorithm against three candidate cache placement schemes, and show via extensive simulation that our algorithm consistently outperforms these alternative schemes.
\end{abstract}

Index Terms-Heuristic optimization, web cache placement, wireless multi-hop networks.

\section{INTRODUCTION}

I N THE LAST few years, there has been an explosive growth of interest in mobile computing, as well as in delivering World Wide Web content and streaming traffic to radio devices. There is a huge potential market for providing palmtops, laptops, and personal communication systems with access to airline schedules, weather forecasts, or location dependent information, just to name a few. Multi-hop ad hoc wireless networks can be used to provide radio devices with such services, anywhere

Manuscript received September 12, 2003; revised October 5, 2004, and September 30, 2005; approved by IEEE/ACM TRANSACTIONS ON NETWORKING Editor V. Padmanabhan. This work was supported in part by the Center for Wireless Communications, San Diego, and by the Italian Ministry for Scientific Research through the PATTERN project.

P. Nuggehalli is with the Center for Electronics Design and Technology, Indian Institute of Science, Bangalore 560012, India.

V. Srinivasan is with the Department of Electrical and Computer Engineering, National University of Singapore, Singapore 117576.

C.-F. Chiasserini is with the Department of Electronics, Politecnico di Torino, 10129 Torino, Italy (e-mail: chiasserini@ polito.it).

R. R. Rao is with the Department of Electrical and Computer Engineering, University of California at San Diego, La Jolla, CA 92093-0436 USA.

Digital Object Identifier 10.1109/TNET.2006.882863 and anytime. Multi-hop ad hoc networks enable users to spontaneously form a flexible communication system. They allow users to access the services offered by the fixed network through multi-hop communications, without requiring infrastructure in the user proximity. However, in order to offer high quality and low cost services to the network nodes, several technical challenges still need to be addressed [1]. First, wireless networks are plagued by scarcity of communication bandwidth; therefore, a key issue is to satisfy user requests with minimum service delay. Second, since network nodes have limited energy resources, the energy expended for transferring information across the network has to be minimized.

In this work, we focus on caching as an attractive technique to efficiently meet these challenges. Two straightforward caching approaches are as follows. The first approach employs a centralized server to deliver information to the network nodes when they require it. The drawback is that the access latency experienced by the nodes, i.e., the time elapsed from the moment a node requests a data item until that item is received, may be considerable due to the multi-hop nature of information transfer. Moreover, both the server load and the network traffic increase with the increase in the number of requests. The second solution consists in placing a copy of the server content onto all network nodes. The advantage of such an approach is that it minimizes access latency, besides decreasing the radio channel contention and the network traffic load [2], [3]. The drawback is that distributing the server content to all nodes implies a high overhead cost for the nodes that transmit and receive such information. It is easy to see that, whenever access latency and overhead cost of data transfer are high, the best approach is to cache the requested information at a limited number of nodes distributed across the network. Caching, in fact, allows us to optimally trade-off between access latency and system overhead cost.

In our study, we address the problem of cache placement. We consider a wireless ad hoc network with $V$ nodes and assume that the network comprises a server where the information $(I)$ needed by the users is originally stored. The information $I$ changes every $T$ units of time and needs to be updated. By anticipating that the user nodes will demand $I$, the server has to determine at which network nodes $I$ should be cached, in order to optimize system performance. We assume stationary or nomadic network nodes. A couple of examples come to mind: users in an airport lobby equipped with laptops and participating in an ad hoc network, wishing to download news or travel information; or wireless IP routers, which enable the instant deployment of multi-hop ad hoc networks for applications ranging from Internet access to temporary wireless LANs. To find an efficient caching strategy, we formulate the problem as follows. Distributing information to the 
network nodes implies a certain overhead cost, which increases with the number of cached nodes. Every node is associated with a service demand, which must be served by a cache. A node receiving service from a cache incurs a cost that depends on its distance from the cache and on the node's demand. Such a cost takes into account both the access latency and the caching overhead cost for the node to access the cache. The objective is to find the optimal cache placement which minimizes the total cost. As we will show later, the problem we have posed is equivalent to a special case of the connected facility location problem [5]-[7], which is known to be NP-hard.

We propose a greedy algorithm, called Efficient Caching Heuristic Optimization (ECHO) algorithm, which provides a suboptimal solution to the cache placement problem. ECHO has the following desirable properties: 1) it is a polynomial time algorithm; 2) it applies to any arbitrary network topology; and 3) it can be implemented in a distributed and asynchronous fashion. The algorithm finds the optimal solution in the case of tree topologies, while it provides an approximate solution for an arbitrary topology. To evaluate the performance of the proposed algorithm, we derive a bound on its performance. We show that ECHO always provides a solution that is within a factor of 6 of the optimal solution. Although this bound is worse than the best known approximation of 3.55 [7], that advantage of our algorithm is that it can be implemented in a distributed fashion. Then, we compare the behavior of ECHO against three simple caching strategies, namely, no-caching, depth caching, and flooding. Numerical results obtained through extensive simulations show that ECHO significantly outperforms these three alternative schemes.

The rest of the paper is organized as follows. Section II reviews previous work on caching strategies for wired as well as wireless networks. In Section III, we present the network model considered in this study and formulate the cache placement problem as a linear programming problem. In Section IV, we describe our distributed greedy algorithm, the so-called ECHO. Through examples, we compare ECHO to the optimal solution in Section $\mathrm{V}$, and derive a bound on its performance in Section VI. Section VII discusses the features of the proposed scheme, while Section VIII describes our simulation setup and presents some numerical results. Finally, Section IX concludes the paper and points at some aspects that will be the subject of future research.

\section{RELATED WORK}

The problem of data replication and caching has been widely studied in the context of wired networks. In [8], the authors address the problem of proxies placement and employ dynamic programming to determine the optimal placement. They consider, however, the case of networks with a tree topology only. A similar approach is used in [9]. This work describes a distributed algorithm which solves the allocation of electronic content over a distribution tree, when storage cost is considered. A solution to the placement problem, which minimizes the data transfer cost for tree topologies, is also proposed in [10]. More general network topologies are considered in [11], where algorithms for Web server placement are presented. The problem addressed there is, however, significantly different from ours. The goal of [11] is to minimize the cost for clients to access copies of the server information, thus it neglects the cost of distributing cache copies to the network nodes. Moreover, the maximum number of copies that can be created is restricted to a fixed value.

In the context of wireless networks, a cooperative cache management scheme for a cellular environment is proposed in [12]. The authors present a simulation study of the problem of cache replication at the base stations, for the case of streaming services. An ad hoc network scenario is considered in [13]. The focus there is on a distributed application software, which implements cooperative Web caching among mobile terminals. Strategies for cache management are also studied with the objective of minimizing energy consumption and network load. Ref. [14] analyzes the performance of various data allocation methods, by focusing on the communication cost between a mobile computer and a stationary computer storing all data.

The works closest to ours are presented in [6] and [7], which propose a centralized approximation algorithm to solve the connected facilities location problem. This can be seen as a more general formulation of our cache placement problem. Our work differs from [6] and [7] in specifically modeling a multi-hop wireless network, and, even more importantly, in proposing a distributed algorithm.

\section{SYSTEM MODEL}

Let $G=(\mathcal{V}, \mathcal{E})$ be a connected graph representing the multi-hop wireless network composed of $V(V=|\mathcal{V}|)$ nodes connected by $E(E=|\mathcal{E}|)$ links. Assume that the network has a static topology and one of the nodes is the network server with an interface to the wired network. As an example, consider the network nodes to be nomadic users equipped with laptops, or nomadic wireless IP routers forming an ad hoc network. Then, suppose that there is some information $(I)$ which is changing every $T$ units of time. The server node can disseminate $I$ to the other nodes via multi-hop routes. During a $T$ unit interval, a node $k$ desires $I$ with probability $p_{k}, 1 \leq k \leq V$. In order to reduce node access latency, at the beginning of a $T$ unit interval, the server caches $I$ at a few nodes in the network. We call this the dissemination phase. We define a cache placement strategy by the vector $Z=\left(z_{1}, z_{2}, \ldots, z_{E}\right)$, where $z_{e}=1$ if a copy of $I$ is transmitted on edge $e \in \mathcal{E}$ during the dissemination phase, and $z_{e}=0$ otherwise. At the end of the dissemination phase, nodes desiring $I$ access the cache location corresponding to the minimum access cost. We call this the access phase.

\section{A. Model Assumptions}

1) Links are bidirectional.

2) The server always possesses a cached copy of $I$.

3) The overhead cost due to transmitting and receiving $I$ along any link is constant and is equal to 1 .

4) We model the access latency cost for a node $k$ $(k=1, \ldots, V)$ by the minimum number of hops required to reach a cached copy of $I$.

5) We assume that storage costs are insignificant.

Note that if we consider the overhead cost as energy, assumption 3 in this context (the energy required to transmit and receive $I$ along any link is constant and is equal to 1) is justified as follows. In wireless technologies, such as IEEE 802.11b, a communication node participating in the network activity can be in 
one of the following operational states: transmitting, receiving, or idle. A node always listens to the channel, and the values of power consumption while receiving or idling are very similar. This implies that listening to an idle or a busy channel lead to the same energy cost. As for transmission, whenever a node wishes to send a packet, once it gains access to the channel, the 802.11 CSMA/CA MAC protocol ensures that there is almost no interference. Furthermore, stationary or nomadic network users imply that fast fading phenomena due to mobility can be ignored. This in turn allows us to assume that the wireless channel is time-invariant. Note also that, in existing multi-hop wireless systems such as IEEE $802.11 \mathrm{~b}$ WLAN and IEEE 802.15 (Bluetooth) WPAN, there is no provision for dynamic power control [15], [16]. In other words, power levels cannot be changed on the fly.

With regard to assumption 4, the linear relationship is motivated by the following. Previous work [17] has shown that delay depends strongly on the number of hops (in fact, it is shown to increase exponentially with hop count). In our model, we have assumed a linear dependence as a first approximation, and for analytical tractability; however, some results obtained when the access latency is a nonlinear function of the hop count (please see Section VIII).

Finally, assumption 5 implies that nodes transmitting a copy of $I$ during the dissemination phase retain $I$ in their buffers. Thus, the terminal nodes of any edge $e$ over which $I$ is transferred (i.e., with $z_{e}=1$ ) posses a copy of $I$. It follows that the subgraph induced by $Z$ is a connected subgraph containing the server. Assumption 5 is motivated by the low cost of memory.

\section{B. Calculation of Costs}

Let $\mathcal{C}$ denote the set of cached nodes and $d_{k}, k=1, \ldots, V$, be the minimum distance in hops from any node $k$ to $\mathcal{C}$. Since the overhead cost of transferring $I$ over each link is the same, the overhead cost incurred during the dissemination phase is given by $K_{\text {diss-overhead }}=\sum_{e \in \mathcal{E}} z_{e}$. The cost incurred during the access phase includes: (i) the overhead cost for the nodes to retrieve $I$ from a cache, and (ii) the access latency that the node experiences. For a strategy $Z$, the expected access overhead cost, $K_{\text {acc-overhead }}$, as well as the expected access latency cost, $K_{\text {latency }}$, are given by $\sum_{k \in \mathcal{V}} p_{k} d_{k}$. Therefore, the average overhead cost and the average access latency experienced by the network system are given by

$$
\begin{aligned}
K_{\text {overhead }} & =K_{\text {diss-overhead }}+K_{\text {acc-overhead }} \\
& =\sum_{e \in \mathcal{E}} z_{e}+\sum_{k \in \mathcal{V}} p_{k} d_{k} \\
K_{\text {latency }} & =\sum_{k \in \mathcal{V}} p_{k} d_{k} .
\end{aligned}
$$

As an example, assume that in the dissemination phase the server caches $I$ at all the nodes in the network. Then, during the access phase, each node will experience zero access latency and zero overhead cost. However, the network ends up wasting valuable resources in relaying $I$ to those nodes who do not desire $I$ in the access phase of a particular interval. On the other hand, if, in the dissemination phase, the server decides not to cache $I$ in any of the other nodes in the network, access latency and overhead costs will be substantial in the access phase.

We define the total cost as

$$
K_{\text {tot }}=K_{\text {overhead }}+\lambda K_{\text {latency }}=\sum_{e \in \mathcal{E}} z_{e}+(1+\lambda) \sum_{k \in \mathcal{V}} p_{k} d_{k}
$$

with $\lambda$ indicating the relative importance of access latency and overhead cost. Notice that, by varying $\lambda$, we can model different network scenarios and quality of service requirements.

Our objective is to find a strategy $Z$ which minimizes the total cost, thus providing the optimal tradeoff between the average overhead cost and the average access latency.

\section{Problem Formulation}

Here, we provide an integer linear programming (LP) formulation for the problem described above. Let $c_{k e}$ be the minimum distance in hops from a node $k$ to an edge $e$. In other words, $c_{k e}$ is the minimum of the shortest path distance between node $k$ and the terminal nodes of edge $e$. Observe that $c_{k e}$ also represents the access latency cost for node $k$ to access $I$ from the nearest terminal node of edge $e$. For ease of formulation, we augment graph $G$ by attaching a virtual node $v$ to the server via an edge $e_{v}$, and, without loss of generality, we refer to the server node as node 1. By virtue of Assumption 2, we have $z_{e_{v}}=1$, for any strategy $Z$.

Consider the following LP formulation.

$$
\begin{aligned}
& \mathbf{P}: \text { Minimize } \quad \sum_{k \in \mathcal{V}} \sum_{e \in \mathcal{E}} p_{k} c_{k e} x_{k e}+M \sum_{e \in \mathcal{E}} z_{e} \\
& \text { s.t. } \quad \sum_{e \in \mathcal{E}} x_{k e} \geq 1 \quad \forall k \in \mathcal{V} \\
& z_{e}-x_{k e} \geq 0 \quad \forall k \in \mathcal{V}, e \in \mathcal{E} \\
& \sum_{e \in \delta(\mathcal{S})} z_{e}-\sum_{e \in \mathcal{S}} x_{k e} \geq 0 \quad \forall \mathcal{S} \subseteq \mathcal{E}, e_{v} \notin \mathcal{S}, \forall k \in \mathcal{V} \\
& x_{k e}, z_{e} \in\{0,1\} \quad \forall k \in \mathcal{V}, e \in \mathcal{E}
\end{aligned}
$$

where $M=1 /(\lambda+1) ;\left\{x_{k e}\right\}$ is a set of assignment variables such that $x_{k e}=1$ if node $k$ accesses information $I$ from the nearest terminal node of edge $e$, else $x_{k e}=0 ; z_{e}=1$ if $I$ is disseminated along edge $e$. From (3), it is easy to see that solving (4) is equivalent to minimizing $K_{\text {tot }}$. Constraint (5) ensures that each node is assigned to at least one cache location from which it can access $I$. Constraint (6) ensures that each node is assigned to an edge whose terminal nodes possess $I$. Constraint (7) is a connectivity constraint on the subgraph induced by strategy $Z$. In particular, $\delta(\mathcal{S})$ represents the cut-set of $\mathcal{S}$ (set of edges adjacent to edges in $\mathcal{S}$ but not belonging to $\mathcal{S}$ ). If $\sum_{e \in \mathcal{E}} x_{k e}=$ 1 for any $k \in \mathcal{V}$, then each node is assigned to exactly one edge, and constraint (7) ensures connectivity of the subgraph induced by $Z$. If, however, a node is assigned to multiple edges, then constraint (7) ensures that each of these edges has an edge disjoint path to $e_{v}$ on the subgraph induced by $Z$. Note that in the optimal solution, each node $k$ will be assigned to only one edge $e$; otherwise, the objective function in (4) could be further optimized. 
As already mentioned in Section I, our optimization problem is a special case of the connected facility location problem, called the rent-or-buy problem, whose formulation is as follows [6]. An existing facility is given, along with a set of locations at which further facilities can be built. Every location $k$ is associated with a service demand, denoted by $p_{k}$, which must be served by one facility. The cost of serving $k$ by using facility $j$ is equal to $p_{k} c_{k j}$, where $c_{k j}$ is the cost of connecting $k$ to $j$. The connection cost of a location hosting a facility is zero. Besides selecting the sites to build the facilities, we also want to connect them by a Steiner Tree [18]. Connecting the facilities incurs a cost which is proportional to the weight of the Steiner Tree. The objective is to find a solution (i.e., to select the locations to build facilities and connect them by a Steiner Tree) which minimizes the total cost. It is easy to see that, in our setting, the facility that is already available represents the server node, new facilities correspond to caches, while locations correspond to the network nodes at which caches (i.e., facilities) can be created.

The rent-or-buy problem is NP-hard [6], [7], however, several approximation algorithms have been developed. A $\rho$-approximation algorithm is defined as a polynomial time algorithm that always finds a feasible solution with an objective function value within a factor of $\rho$ of the optimal cost. The best approximation algorithm known today for the rent-or-buy problem was developed by Gupta et al. [7], who gave a 3.55-approximation algorithm. The solution in [7] requires a centralized implementation, therefore, it is not suitable for a multi-hop wireless environment. In the following, we design a polynomial-time algorithm, that approximates the optimal solution for any arbitrary graph within a factor of 6 . Our algorithm allows for a distributed and asynchronous implementation.

\section{A GREEDY SOLUTION}

We propose a greedy algorithm, called ECHO, which provides an approximate solution to problem $\mathbf{P}$ in (4). ECHO decides which nodes should cache $I$ during the dissemination phase, and is such that it can be implemented in a distributed and asynchronous manner. To motivate our scheme, we first consider the special case of tree topologies, then we describe the algorithm for the general case of connected graphs.

\section{A. A Special Case: Tree}

For a node $k$, let $f(k)$ be the number of progeny of $k$ (i.e., all descendent nodes of $k$ ) given by $s_{1}, s_{2}, \ldots, s_{f(k)}$. Let $s_{0}$ be the node $k$ itself. Clearly $f(1)=V-1$, since node 1 is the server. Suppose node $k$ 's parent node (say $m_{k}$ ) is a cached node. If $m_{k}$ disseminates $I$ to $k$, it will incur an additional overhead cost of 1 unit (see Assumption 3). However, $k$ and its progeny will experience a decrease in access latency and overhead cost given by $(1+\lambda) \sum_{j=0}^{f(k)} p_{s_{j}}$. Therefore, $m_{k}$ will disseminate $I$ to $k$ if

$$
(1+\lambda) \sum_{j=0}^{f(k)} p_{s_{j}} \geq 1
$$

i.e.,

$$
\sum_{j=0}^{f(k)} p_{s_{j}} \geq M
$$

This dissemination strategy can be implemented in polynomial time and, in the special case of a tree topology, it can be easily shown by construction to be optimal. In general, however, the optimal solution is not unique. When $\sum_{j=0}^{f(k)} p_{s_{j}}=M$, the cost of the solution remains the same, irrespective of $m_{k}$ 's decision.

Assume that $I$ is cached at node $k$. Let $c_{1}, c_{2}, \ldots, c_{l}$ be the children of node $k$. Since the graph is a tree, the progeny of $c_{i}$, $1 \leq i \leq l$ are disjoint sets. Thus, the decision to cache at node $c_{i}$ can be made independently of the decision with respect to node $c_{j}, i \neq j$. The disjointedness of progeny is a desirable property, since it lends itself to an asynchronous and distributed implementation of our algorithm; however, this property does not hold for a general graph. In the next section, we extend the algorithm described here for a tree topology to the case of networks with a generic topology, in such a way that the progeny sets are disjoint.

\section{B. The General Case}

We now describe the dissemination algorithm in the case of a network with a generic topology.

For ease of description, we divide the algorithm into stages. We say that an edge is open if its terminal nodes are chosen as caches. Initially, all edges are closed, except for $e_{v}$. An edge $e$ is said to be a neighbor of an edge $l$ (denoted by $e \perp l$ ) if $e$ and $l$ share a common node. Let $\mathcal{O}$ be the set of opened edges. Let $\mathcal{N}_{e}$ be the set of neighbor edges of edge $e$. At any stage $n$, let $\mathcal{B}_{e}$ be the set of neighbor edges of $e$, which have not been opened yet $\left(\mathcal{B}_{e} \subseteq \mathcal{N}_{e}\right)$.

We associate a variable $\alpha_{k}$ with each node. At each stage $n$, $\alpha_{k}$ can be viewed as the price that node $k$ is willing to pay to any available cache to access $I$. We say that node $k$ is tight with an edge $e$ if $\alpha_{k} \geq c_{k e} p_{k}$. When a node goes tight with a cache, it implies that the price the node pays compensates for the cost incurred by the cache in disseminating $I$ to the node. A node can be in two states: frozen and unfrozen. We say that a node $k$ is frozen when we stop increasing the associated variable $\alpha_{k}$. When a node is frozen, it cannot become tight with any new edge.

For each edge $e$, we define $\mathcal{R}_{e}$ as the set of nodes, who belong to the progeny of the terminal nodes of edge $e$, and receive their copy of $I$ either through $e$ or one of its descendent edges. Note that $\mathcal{R}_{e_{v}}=\mathcal{V} \backslash\{1\}$. Let $\mathcal{F}_{n}$ be the set of edges opened at stage $n$. Let $\mathcal{T}_{e}$ be the set of nodes tight with edge $e$. Then the demand, $D_{e}$, for edge $e$ is given by $\sum_{k \in \mathcal{T}_{e}} p_{k}$.

The algorithm is as follows.

Step 1. Initialize all variables: Set $n=0 ; \mathcal{O}=\left\{e_{v}\right\}$; $\mathcal{B}_{e_{v}}=\mathcal{N}_{e_{v}} ; \alpha_{k}=0, \forall k \in \mathcal{V} ; \mathcal{R}_{e_{v}}=\mathcal{V} \backslash\{1\} ; \mathcal{F}_{-1}=\left\{e_{v}\right\}$.

The algorithm begins by opening edge $e_{v}$, and initializing $\mathcal{B}_{e_{v}}$ to the set of neighbor edges of the server node, i.e., the set of edges along which I can be disseminated.

Step 2. $\mathcal{F}_{n}=\emptyset, \alpha_{k}=0, \forall k \in \mathcal{V}$.

For any $e \in \mathcal{F}_{n-1}$,

Step 2.a. During this step, we shall tentatively open some edges belonging to $\mathcal{B}_{e}$, and freeze all progeny of edge e. 
While all nodes in $\mathcal{R}_{e}, e \in \mathcal{F}_{n-1}$, are not frozen

1. Freeze node $k \in \mathcal{R}_{e}$ if it is tight with edge $e$ or with a tentatively open edge $l \in \mathcal{B}_{e}$.

2. For any edge $l \in \mathcal{B}_{e}$, if $D_{l} \geq M$, then declare $l$ to be tentatively open.

An edge, which is not already open and has at least $M$ units of demand, is tentatively opened. This is because, as highlighted in (10), if I is disseminated along this tentatively open edge, then the decrease in access cost outweighs the increase in dissemination cost.

3. Define $\tilde{\mathcal{R}}_{e}=\left\{k: k \in \mathcal{R}_{e}, k\right.$ not frozen $\}$

Increase $\alpha_{k}$ for all nodes in $\tilde{\mathcal{R}}_{e}$ by

$\min _{\left\{k \in \tilde{\mathcal{R}_{e}}, l \in \mathcal{B}_{e} \cup\{e\}\right\}}\left(c_{k l} p_{k}-\alpha_{k}\right)$.

Variables $\alpha_{k}$ 's are raised by the minimum amount which is sufficient to let some node $k \in \tilde{\mathcal{R}}_{e}$ become tight with some edge $l \in \mathcal{B}_{e} \cup\{e\}$. Notice that many nodes may become tight at the same time.

End While

Step 2.b. Let $\mathcal{L}_{e}$ be the set of tentatively open edges in $\mathcal{B}_{e}$.

If $\mathcal{L}_{e}=\emptyset$

Stop dissemination beyond edge $e$.

Else

Let $\mathcal{H}_{l}, l \in \mathcal{L}_{e}$ be the set of nodes tight with tentatively open edge $l$.

Let $\mathcal{H}$ be the collection of sets $\left\{\mathcal{H}_{l}: l \in \mathcal{L}_{e}\right\}$.

At this step, a further optimization is achieved as follows. Suppose $l_{1}$ and $l_{2}$ are two tentatively open edges originating from the same parent edge. Suppose that edge $l_{1}$ has $n_{1}$ nodes tight with it and node $l_{2}$ has $n_{2}<n_{1}$ nodes tight with it. Also let the number of nodes which are tight with both $l_{1}$ and $l_{2}$ be $n_{c}$. If we disseminate to edge $l_{1}$, then the number of additional nodes which edge $l_{2}$ can serve is $n_{2}-n_{c}$. If the demand created by these $n_{2}-n_{c}$ nodes is less than $M$, then disseminating to edge $l_{2}$ is suboptimal. The operations described in the following avoid such redundant edges from being opened.

While $\mathcal{H} \neq \emptyset$

$$
\begin{aligned}
& l^{*}=\arg \max _{l \in \mathcal{L}_{e}} \sum_{k \in \mathcal{H}_{l}} p_{k} \\
& \mathcal{Q}_{l^{*}}=\mathcal{H}_{l^{*}}
\end{aligned}
$$

For each $\mathcal{H}_{l} \in \mathcal{H}$

$$
\begin{gathered}
\mathcal{H}_{l}=\mathcal{H}_{l} \backslash \mathcal{H}_{l^{*}} \\
\text { If } \sum_{k \in \mathcal{H}_{l}} p_{k}<M \\
\mathcal{H}=\mathcal{H} \backslash \mathcal{H}_{l}
\end{gathered}
$$

End If
End While

$$
\begin{aligned}
& \text { Let } \mathcal{A}=\left\{l: l \in \mathcal{L}_{e}, \sum_{k \in \mathcal{Q}_{l}} p_{k} \geq M\right\} . \\
& \mathcal{O}=\mathcal{O} \bigcup \mathcal{A}, \mathcal{F}_{n}=\mathcal{F}_{n} \bigcup \mathcal{A}, \mathcal{R}_{l}=\mathcal{Q}_{l} .
\end{aligned}
$$

End If

\section{End For}

For any $e \in \mathcal{F}_{n}, \mathcal{B}_{e}=\left\{l: l \in \mathcal{N}_{e}, l \not \perp l^{\prime} \forall l^{\prime} \in \mathcal{O} \backslash\{e\}\right\}$

Further let $k_{l}, l \in \mathcal{B}_{e}$, be the terminal node of $l$ which is not cached. Then, $\mathcal{B}_{e}=\mathcal{B}_{e} \bigcap\left\{l: k_{l} \in \mathcal{R}_{l}\right\}$

$\mathcal{B}_{e}$ is updated with all neighbor edges of e which are not already opened and are not neighbors of any open edge, other than $e$.

Step 3. If $\mathcal{F}_{n} \neq \emptyset, n=n+1$ and go to Step 2 .

Otherwise, terminate algorithm.

We observe that the above algorithm allows us to create disjoint progeny sets. In fact, at the end of each stage $n$, we have that: $\mathcal{R}_{e} \bigcap \mathcal{R}_{e^{\prime}}=\emptyset, \forall e, e^{\prime} \in \mathcal{F}_{n}$.

\section{Comparing the Performance of ECHO WITH THE OPTIMAL SOLUTION}

Here, we would like to compare the performance of the proposed algorithm with the optimal solution. Since the optimization problem we have posed is NP-hard, we compute the optimal solution by using the following brute force approach. We list all spanning trees of the graph, and, for each spanning tree, we derive the optimal cache placement using (10). Finally, we choose the spanning tree with the least cost. Clearly, we need to consider simple topologies so that the computation of the optimal solution does not become exceedingly cumbersome. In the following, two examples are reported.

1) Example 1: Consider the topology in Fig. 1, with $\lambda=1$, $p_{k}=0.25, \forall k \in \mathcal{V}$, resulting in $M=0.5$. At the beginning of stage 0 , only the virtual edge, $e_{1}$ is open (only the server has information I), as shown by Fig. 1(a). The progeny list for the server includes all the nodes in the network. Edges $e_{2}, e_{3}$ and $e_{4}$ are the edges belonging to $\mathcal{B}_{e_{1}}$ (i.e., nodes 2,3 , and 4 are potential cache locations). Also, initially we have that all $\alpha_{k}=0$, for $k=1, \ldots 7$. Node 1 becomes frozen with $\alpha_{1}=0$ since it is tight with edge $e_{1}$. Since all access probabilities are the same and equal to $0.25, \alpha_{k}$ 's are increased by 0.25 (see Step 2.a.3). When $\alpha_{k}$ becomes equal to 0.25 , for $k=2, \ldots 7$, nodes 2,3 , and 4 get frozen with edge $e_{1}$, node 5 becomes tight with edge $e_{2}$, node 6 becomes tight with edge $e_{3}$, and node 7 becomes tight with edge $e_{4}$. Since all the edges that could be opened have demand less than $M=0.5$, none of them are tentatively opened. In the next step, $\alpha_{k}=0.5$, for $k=\{5,6,7\}$. As a result, nodes 5 and 6 become tight with edge $e_{2}$, nodes 5,6 , and 7 become tight with edge $e_{3}$ and nodes 6 and 7 become tight with edge $e_{4}$. Since all the edges that could be opened have demand of at least $M=0.5$, they are all tentatively opened and all nodes in the network get frozen. It is easy to see that only edge $e_{3}$ will be opened in Step 2.b.

At the beginning of stage 1 , edges $e_{1}$ and $e_{3}$ are open, as shown in Fig. 1(b). The progeny list for edge $e_{3}$ are nodes 5,

End For 


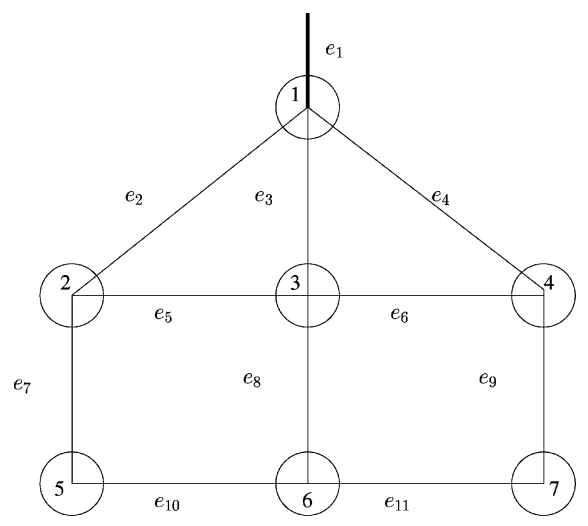

(a)

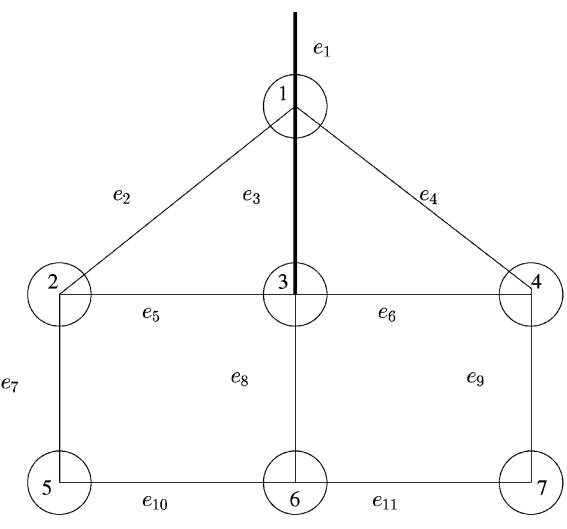

(b)

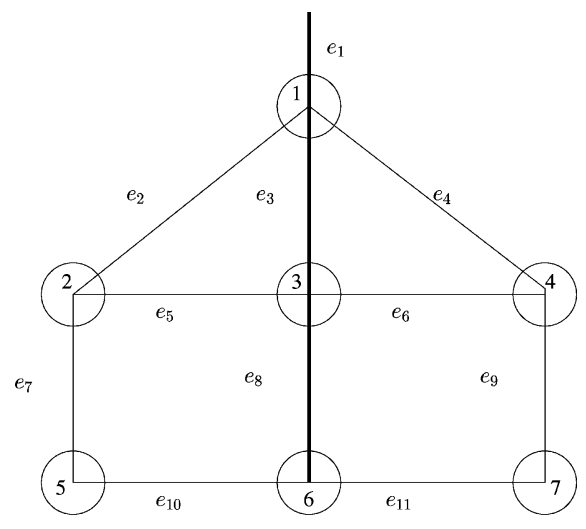

(c)

Fig. 1. Topology for network 1. The thick lines indicate open edges. (a) Stage 1: In the beginning only the virtual edge is open. (b) Stage 2: Edges $e_{1}$ and $e_{3}$ are open. (b) Stage 3: Edges $e_{1}, e_{3}$ and $e_{8}$ are open.

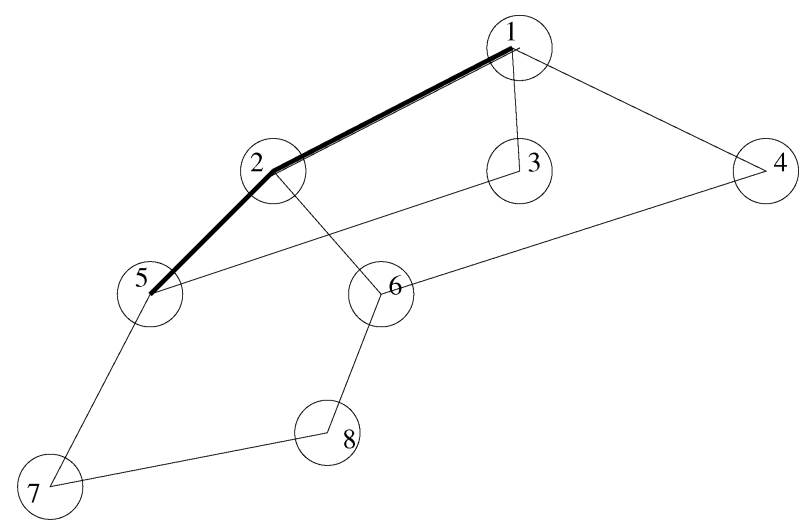

Fig. 2. Topology for network 2 . The thick lines indicate open edges.

6 , and 7. The edges that can be opened are $e_{5}, e_{6}$ and $e_{8}$. It is easy to see that of these edges only edge $e_{8}$ will be opened. The algorithm then terminates with the cache locations as shown by Fig. 1(c).

In this example, it turned out that this cache placement is also the optimal one. However, if $\lambda=0$, ECHO results in the cache location shown in the middle diagram in Fig. 1, while the optimal strategy would be not to cache at all.

2) Example 2: Here we aim at showing the computational savings gained by using our algorithm. We consider the topology shown in Fig. 2. As before, we assume $\lambda=1$, $p_{k}=0.25, \forall k \in \mathcal{V}$, thus resulting in $M=0.5$. In this case, it can be seen that ECHO achieves the optimal cache placement with a computation effort that is polynomial in the size of the graph. While to compute the optimal solution for the same topology, we need to consider 135 spanning trees. Clearly, for larger networks, the number of spanning trees becomes astronomically large.

\section{ANALYSIS}

In this section, we provide a bound on performance for the ECHO algorithm. In Section IV-A, we noticed that the ECHO algorithm achieves the optimal value for trees. Now, we show that, for an arbitrary topology, our algorithm approximates the optimal solution within a factor of 6 .
Recall from Section III-C that we obtain a cache placement strategy $Z$ by setting $z_{e}=1$ for all the open edges. We can prove the following property.

Lemma 1: The graph induced by the strategy $Z$, obtained from the ECHO algorithm, is a tree.

Proof: By induction. At any stage $n$ of the algorithm, consider the set of edges which are opened at the end of the stage. The only way a cycle can be formed is if a tentatively opened edge forms a cycle with already opened edges, or two tentatively opened edges which are neighbors are opened simultaneously. The former can never happen since Step 2 ensures so. The latter also is infeasible since the sets of progeny are disjoint.

We have the following theorem.

Theorem 1: Let $O P T$ be the optimum value of the primal integer problem $\mathbf{P}$ in (4). The solution obtained by the algorithm is at most $6 \times O P T$.

Proof: See the Appendix .

This bound is very close to the best bound in the literature [7] for the optimization problem that we have posed. Ref. [7] gives a 3.55-approximation by using a centralized approach, while our algorithm can be implemented in a distributed and asynchronous fashion.

\section{IMPLEMENTATION OF ECHO}

In order to implement ECHO, each node needs to maintain some progeny and distance information. This kind of information is readily available from current routing mechanisms [19]. We show in this section that the ECHO algorithm can be implemented for a network with arbitrary topology in a distributed and asynchronous manner.

Consider the set of open edges at the end of stage $n$. For simplicity, let $e_{1}$ and $e_{2}$ be the only two edges which were opened in stage $n$. Let $G_{1}$ and $G_{2}$ be the subgraph of $G$ induced by the progeny sets $\mathcal{R}_{e_{1}}$ and $\mathcal{R}_{e_{2}}$, respectively. In subsequent stages, caches are opened on edges belonging to $G_{1}$ and $G_{2}$. Opened edges in $G_{1}\left(G_{2}\right)$ will inherit progeny which are subsets of $\mathcal{R}_{e_{1}}\left(\mathcal{R}_{e_{2}}\right)$. From the algorithm description, we know that the progeny of $e_{1}$ and $e_{2}$ are disjoint. This implies that, caching decisions in the subgraphs, $G_{1}$ and $G_{2}$, are independent. The disjointedness of progeny therefore allows for a distributed imple- 
mentation of ECHO. This argument easily extends to the case when multiple edges are opened in a particular stage.

We now show that the ECHO algorithm can be implemented without the need for any synchronization. Consider the situation when a cached node $k$ has to decide to which of its neighbor nodes should $I$ be disseminated. If each of $k$ 's progeny communicates its $\alpha$ value during every stage of the dissemination algorithm, then the number of messages exchanged becomes very large resulting in a large overhead. Moreover, if nodes update their $\alpha$ 's asynchronously, then some errors may occur. For example, it could happen that a node still increases its $\alpha$ after being frozen with an edge. In this case, some edges would be erroneously opened. To avoid such problems, we propose the following scheme. We know that any cached node $k$ has knowledge of its immediate neighbors, as well as the progeny set that it serves. Node $k$ multicasts to its progeny the set of potential cache locations. The progeny, in turn, respond with the shortest path distance to each of the potential cache locations that can be opened, as well as their access probabilities. Node $k$ can then locally run the algorithm to determine which of the possible cache locations should be opened. This avoids all timing issues and obviates the need for synchronization in the network. We stress that by distributed we refer to the fact that each node takes independent decisions to propagate the cache. ECHO does need global topological information and disseminating such information in a low-cost manner, i.e., bandwidth and energy efficient manner, is a challenging problem. However, efficient means of route discovery have been devised [19] which are beyond the scope of our work. Also, this routing burden is imposed only when the topology changes, not every time the information is updated.

\section{NUMERICAL RESULTS}

In this section, we present some results showing the sensitivity of the placement algorithm to the system parameters introduced in the previous sections. The performance of the proposed algorithm is compared with the results obtained using three simple schemes, namely no-caching $(N C)$, depth caching $(D C)$, and flooding $(F L D)$. In the NC scheme, $I$ is stored at the server only. The DC scheme corresponds to disseminating $I$ to all nodes that are up to $h$ hops away from the server. Parameter $h$ is chosen heuristically as a function of the access probabilities and $\lambda$ : as $\lambda$ increases, so does $h$. Finally, in the FLD scheme, $I$ is disseminated to all the nodes in the network.

We construct random topologies by choosing $V=30$ random points in a square of unit area. Each node is assumed to have a communication range of $D_{\max }$, which is taken as a varying parameter of the system. Given the set of random points and $D_{\max }$, one can construct the graph $G=(\mathcal{V}, \mathcal{E})$, where two nodes are connected by an edge if their distance is less than $D_{\max }$. We ensure that the resulting graph $G$ is connected. If not, we generate another topology until we get a connected graph. One of the nodes is arbitrarily identified as the server. We consider two scenarios: one with uniform access probabilities $\left(p_{k}\right.$ 's), the other with nonuniform access probabilities.

We first compare the performance of ECHO versus NC, FLD and DC by fixing $\lambda$ at $1, D_{\max }$ at 0.3 , and the value of the access
TABLE I

COMPARISON OF CACHING STRATEGIES AVERAGED OVER 30 RUNS, FOR $V=30, D_{\max }=0.3$, UNIFORM ACCESS PROBABILITIES EQUAL to $1 / 6$ AND $\lambda=1$

\begin{tabular}{|c|c|c|c|}
\hline & Caching overhead & Access latency & Total cost \\
\hline NC & 13.5667 & 13.5667 & 27.1333 \\
FLD & 29 & 0 & 29 \\
DC & 20.2833 & 6.9167 & 27 \\
ECHO & 11.9889 & 5.3222 & 17.311 \\
\hline
\end{tabular}

\section{TABLE II}

COMPARISON OF CACHING STRATEGIES AVERAGED OVER 30 RUNS, FOR $V=30, D_{\max }=0.3$, ACCESS ProbabiLITIES EQUAL TO: $1 / 4,1 / 6,1 / 9$, AND $\lambda=1$

\begin{tabular}{|c|c|c|c|}
\hline & Caching overhead & Access latency & Total cost \\
\hline NC & 14.0741 & 14.0741 & 28.1482 \\
FLD & 29 & 0 & 29 \\
DC & 25.7787 & 5.8120 & 31.5907 \\
ECHO & 12.2787 & 5.6787 & 17.9574 \\
\hline
\end{tabular}

probabilities. The comparison is obtained by averaging the results over 30 different topologies. The performance is derived in terms of average overhead cost, access latency, and total cost, as defined in (1)-(3). Table I shows the results for the case where all access probabilities are the same and equal to $1 / 6$, while Table II presents the results when we have nonuniform access probabilities. Nonuniform access probabilities are set as follows; we divide the 30 nodes into three groups of equal size, and assign to each of them a different value of access probability (namely, $1 / 4,1 / 6$, and 1/9).

As expected, the results presented in the two tables show that the highest overhead cost is obtained with the FLD scheme, while the highest access latency is given by the $\mathrm{NC}$ scheme. However, it is interesting to notice that the FLD and the NC scheme have almost the same total cost in both the scenarios. Also, observe that the overhead cost for the NC scheme is greater than zero, since we have to take into account the overhead cost for the nodes to access $I$ from the server. The DC algorithm has access latency performance close to ECHO, but it implies a much higher overhead burden. Finally, compared to the other three schemes, ECHO allows for a reduction in the total cost ranging between $36 \%$ to $43 \%$.

Next, we investigate the impact of $\lambda$ on performance, when $D_{\max }$ is equal to 0.3 and the access probabilities are all the same and equal to $1 / 6$. Figs. $3-5$ show the values of access latency, overhead burden and total cost as $\lambda$ varies, for a fixed network topology. Looking at the plots, we see that ECHO consistently outperforms the other three caching strategies. (The only exception is for the FLD scheme, which always gives zero access latency as shown in Fig. 3.) For instance, for $\lambda=1$, ECHO gives a reduction in the total cost of $38 \%$ with respect to DC and of 50\% relative to NC. Also, it is interesting to notice that the access latency and overhead plots behave like step functions for ECHO and DC. This is because, for both the schemes, the caching strategy changes only at certain break points. A similar behavior is observed when the access latency for a node is not longer modeled as a linear function of the hop count $(\nu)$ but increases with the number of hops as $\nu^{2}$. The results are presented in Figs. 6-8. We note that the ECHO heuristic still outperforms all other schemes in terms of cost, overhead and delay (the only 


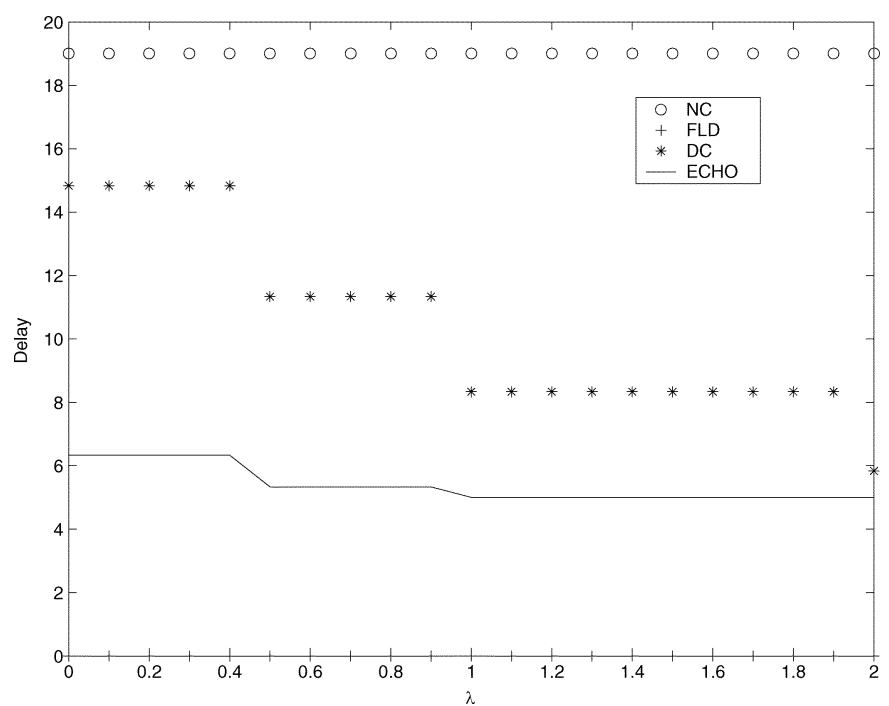

Fig. 3. Access latency versus $\lambda$, for a fixed topology, $V=30, D_{\max }=0.3$, and uniform access probability equal to $1 / 6$.

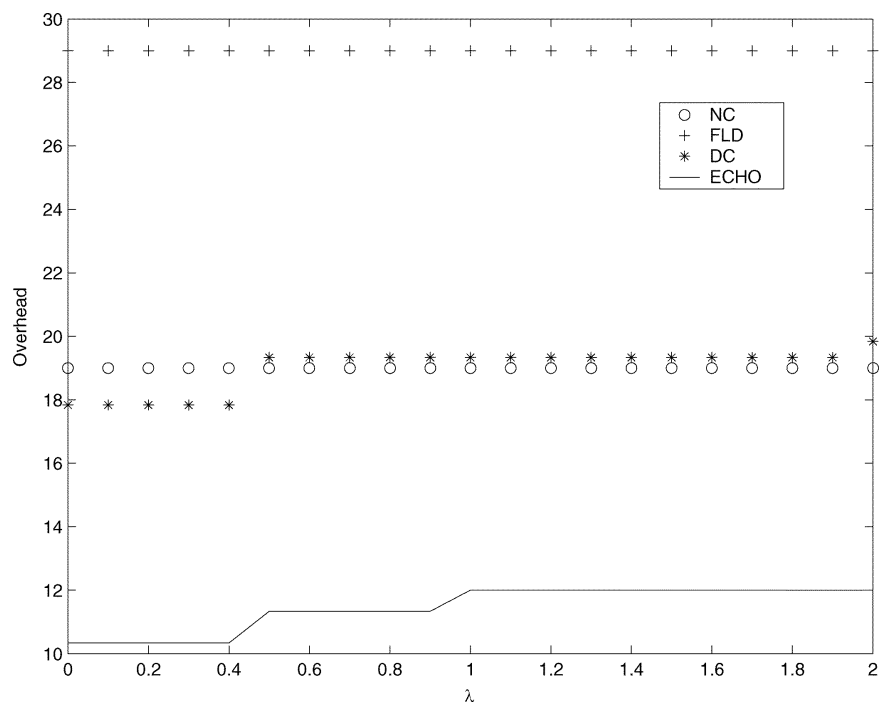

Fig. 4. Caching overhead versus $\lambda$, for a fixed topology, $V=30, D_{\max }=$ 0.3 , and uniform access probability equal to $1 / 6$.

exception is for the flooding scheme as before). As an example, the cost reduction achieved through $\mathrm{ECHO}$ with respect to the DC scheme is between $30 \%$ and $40 \%$, depending on the value of $\lambda$.

Next, we investigate the cost as $D_{\max }$ changes. We consider a fixed topology and assume that the output transmit power increases as $D_{\max }^{\gamma}$, where $\gamma$ is a path loss exponent between 2 and 4. We highlight that, as before, power control is not considered: for a fixed value of $D_{\max }$, all nodes use the same power level, proportional to $D_{\max }^{\gamma}$. As $D_{\max }$ increases, the graph becomes more connected, decreasing access latency but increasing the dissemination overhead and access overhead costs. The results are shown in Fig. 9. As we can see, the optimal operational point (i.e., optimal value of output transmit power) lies somewhere between the minimum power to ensure connectivity and the maximum power. Notice that the curve is not a smooth function. This is because the caching strategy changes only at certain discrete

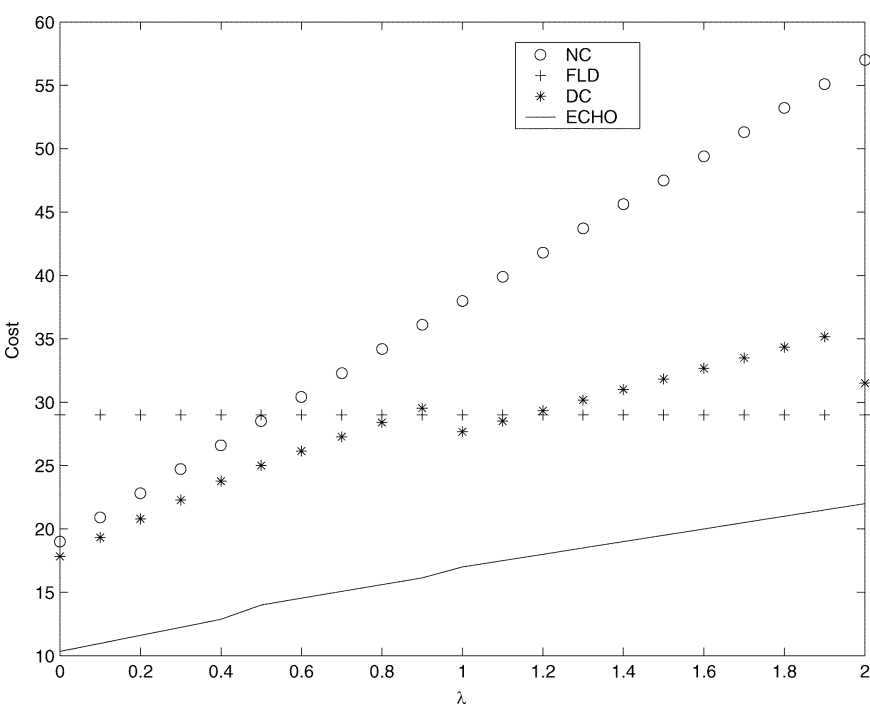

Fig. 5. Total cost versus $\lambda$, for a fixed topology, $V=30$, and uniform access probability equal to $1 / 6$.

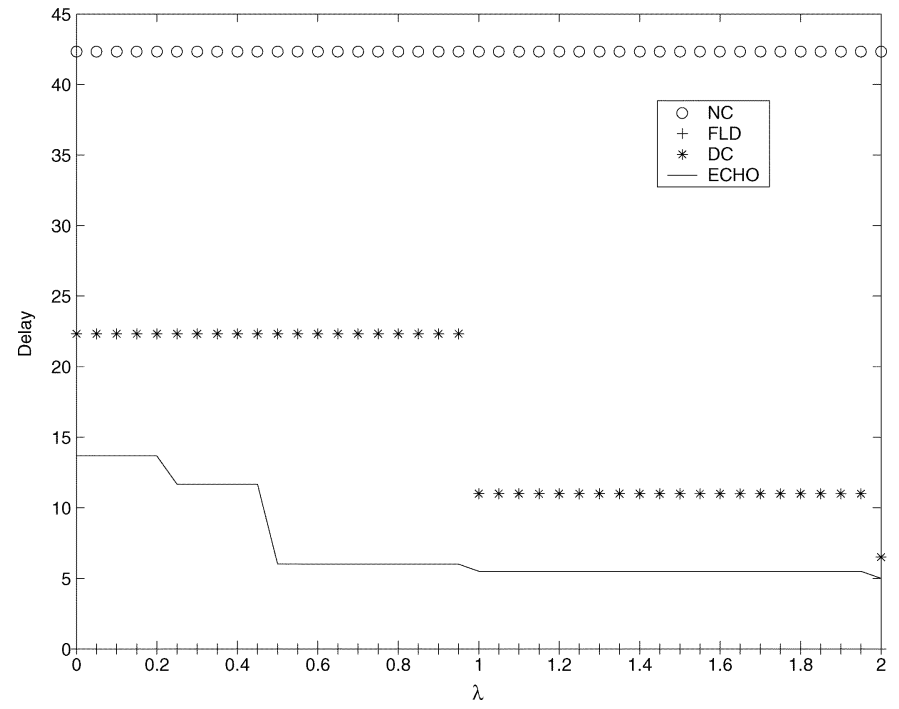

Fig. 6. Access latency versus $\lambda$, for a fixed topology, $V=30, D_{\max }=0.3$, uniform access probability equal to $1 / 6$, and under the assumption that the access latency cost is a nonlinear function of the hop count.

power points. In between these points, the caching strategy remains the same, but since the power is increasing, the overhead cost increases while the access latency cost remains the same, thereby increasing the total cost.

The results above are derived by assuming that in a given $T$ unit interval, all information requests are generated at the same time. We now relax this assumption and show via simulation that, even if requests are generated at different time instants, ECHO gives significantly better performance than other cache placement schemes.

To highlight the difference with respect to our earlier assumption, consider the following situation. Let $C_{1}$ be a cached node with progeny nodes $n_{1}$ and $n_{2}$. Let $n_{2}$ be downstream of $n_{1}$. If node $n_{2}$ generates a request before $n_{1}$ in a $T$ unit interval, then when $n_{1}$ relays $I$ to $n_{2}$, it can keep a copy of $I$ in its cache since memory cost is zero. Therefore, when $n_{1}$ generates a request for 


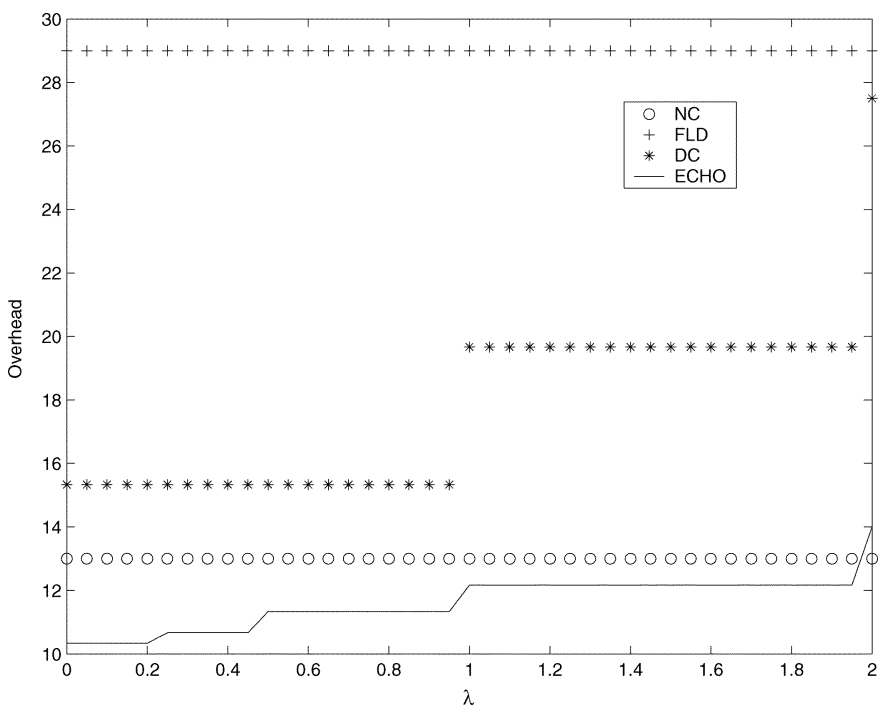

Fig. 7. Caching overhead versus $\lambda$, for a fixed topology, $V=30, D_{\max }=$ 0.3 , uniform access probability equal to $1 / 6$, and under the assumption that the access latency cost is a nonlinear function of the hop count.

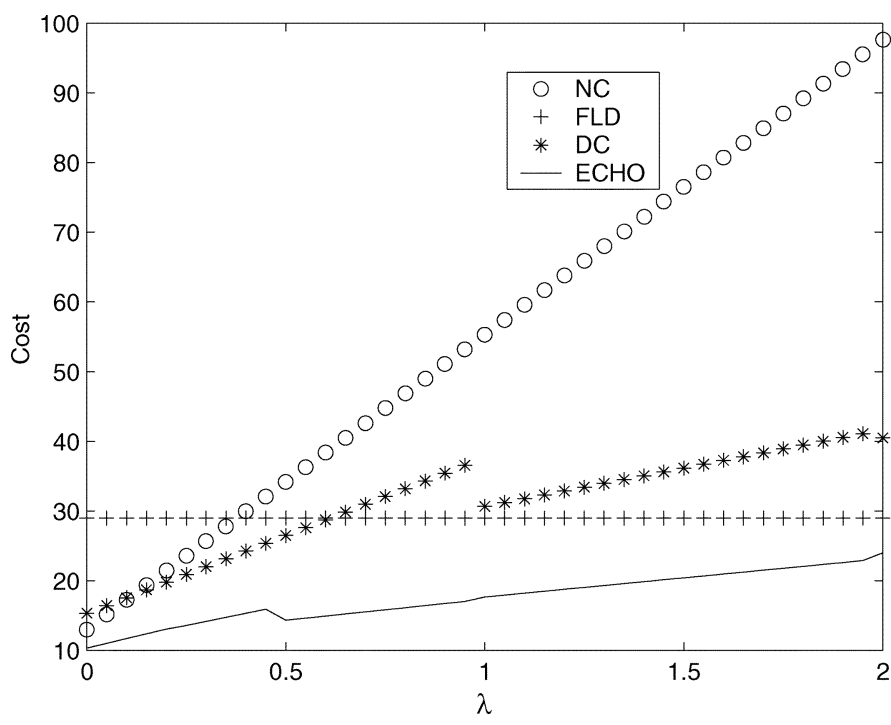

Fig. 8. Total cost versus $\lambda$, for a fixed topology, $V=30$, uniform access probability equal to $1 / 6$, and under the assumption that the access latency cost is a nonlinear function of the hop count.

$I$, the overhead cost and delay cost are zero for $n_{1}$. This example suggests that our earlier formulation overestimates the delay and overhead costs. Thus, one may wonder whether a simple scheme, which involves no a priori caching thereby avoiding any wasteful dissemination, might perform better than ECHO.

To show that ECHO always outperforms other strategies, we consider the following simulation scenario. We set $T$, i.e., the duration for which $I$ remains unchanged, to 10000 . In each $T$ unit interval a node $k$ generates a request with probability $p_{k}$. If $k$ generates a request, then the time at which it generates the request is chosen uniformly between 0 and $T$. The time for transferring a relay request over a hop is equal to 0.001 and the overhead cost is also 0.001 . The time taken for information $I$ to be relayed over one hop is equal to 1 and the associated overhead cost is also 1 unit. We generate 30 different topologies. For each

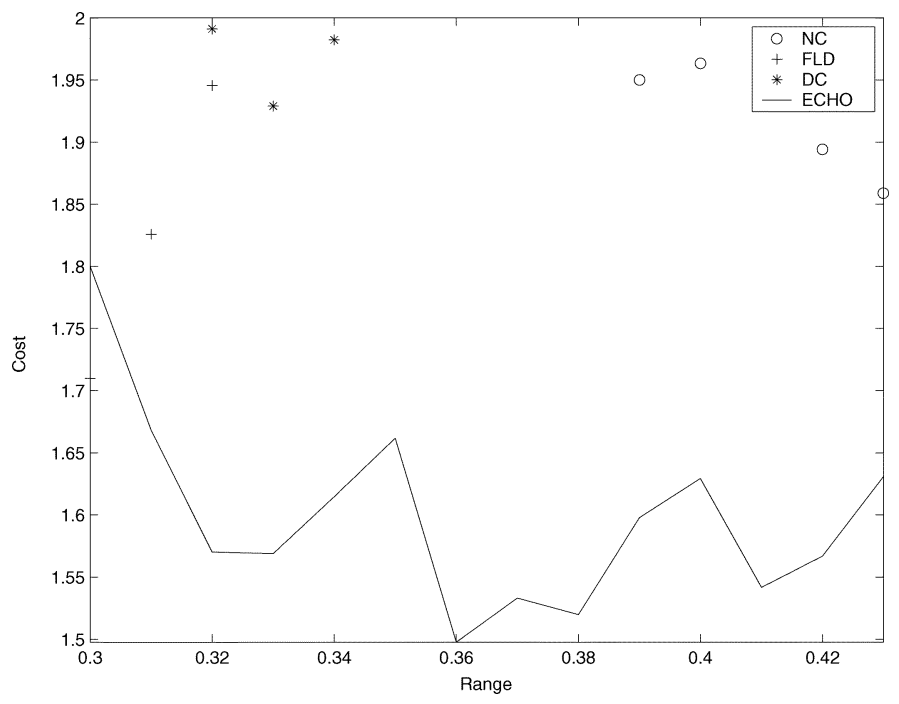

Fig. 9. Total cost versus the nodes radio range $\left(D_{\max }\right)$, for a fixed topology, $V=30$, uniform access probability equal to $1 / 6$, and $\lambda=1$.

TABLE III

COMPARISON OF CACHING STRATEGIES AVERAGED OVER 30 RUNS, FOR $V=30, D_{\max }=0.3$, Access Probabilities EQual to 1/4, 1/6, 1/9, AND $\lambda=1$. INFORMATION REQUESTS ARE GENERATED AT DIFFERENT TIME INSTANTS

\begin{tabular}{|c|c|c|c|}
\hline & Caching overhead & Access latency & Total cost \\
\hline NC & 10.7023 & 10.7023 & 21.4046 \\
FLD & 29 & 0 & 29 \\
DC & 21.7075 & 2.3742 & 24.0817 \\
ECHO & 11.7165 & 4.0831 & 15.7996 \\
\hline
\end{tabular}

topology, we average our results over 1000 iterations. We then average the results over the 30 arbitrarily generated topologies. As before, we divide the 30 nodes into three groups of equal size, and assign to each of them a different value of access probability (namely, 1/4, 1/6, and 1/9).

The results that we obtain in this case are presented in Table III. They show similar performance as the results presented in Table II. However, we notice that the access latency obtained through ECHO is larger than the one obtained by using the DC strategy, although ECHO still gives the minimum total cost. As expected, the cost values that we attain for the $\mathrm{NC}$, the DC and the ECHO schemes are smaller than those derived in the case where all information requests are generated at the same time - an assumption that overestimates the delay and overhead costs.

\section{CONCLUSIONS AND DISCUSSION}

We addressed the problem of optimal cache placement in multi-hop wireless networks. We considered overhead cost and access latency as performance indices. In particular, we defined the system overhead cost to include both the overhead due to the information distribution to various nodes across the network, and the overhead for nodes to access the desired information from the nearest cache. To minimize the weighted sum of overhead cost and access latency, we formulated an integer linear programming problem. This turned out to be an NP-hard problem. Thus, we proposed a polynomial time 
algorithm, which determines at which nodes to place caches so that performance is maximized. Our algorithm has the desirable property that it can be implemented in a distributed and asynchronous fashion, and it applies to any arbitrary network topology. It provides an optimal solution in the case of tree topologies, and an approximate solution in the case of an arbitrary topology. Nevertheless, the bound that we derived on the algorithm performance shows that we always obtain a solution within a factor of 6 of the optimal solution. This is very close to the best approximation known today for the cache placement problem, which can be achieved by employing a centralized approach. Numerical results show that, when compared against three candidate caching schemes, our algorithm significantly outperforms these alternative cache placement strategies.

We would also like to stress that our problem formulation is general enough to include various notions of cost. The cost of each link could account for both energy cost and bandwidth cost.

Also, in our paper $I$ is one piece of information that every one accesses; the work could be extended to the case where multiple pieces of information exist and are accessed with different probability depending on their popularity.

\section{APPENDIX}

Theorem 2: Let $O P T$ be the optimum value of the primal integer problem $\mathbf{P}$ in (4). The solution obtained by the algorithm is at most $6 \times O P T$.

We obtain a caching and access strategy by setting $z_{e}=1$ for all the open edges, and $x_{k e}=1$ for the open edge nearest to $k$ such that $k \in R_{e}$. This ensure that constraints (5) and (6) are met. By using Lemma 1, constraint (7) is also satisfied. An edge $e$ is given by the set $\left\{r_{e}, t_{e}\right\}$, where nodes $r_{e}$ and $t_{e}$ are the terminal nodes of edge $e$. In order to provide a bound, we will construct a dual feasible solution for our primal problem (4). By introducing the dual variables $\beta$ and $\theta$, we obtain the following formulation for the dual problem.

$$
\begin{gathered}
\text { Maximize } \sum_{k \in \mathcal{V}} \alpha_{k} \\
\text { s.t. } \alpha_{k}-\beta_{k e}-\sum_{\mathcal{S} \subseteq \mathcal{E}, e \in \mathcal{S}, e_{v} \notin \mathcal{S}} \theta_{\mathcal{S}, k} \leq c_{k e} p_{k} \quad \forall k \in \mathcal{V}, e \in \mathcal{E} \\
\sum_{k \in \mathcal{V}} \beta_{k e}+\sum_{k \in \mathcal{V} \subseteq} \sum_{\mathcal{S} \subseteq \mathcal{E}: e \in \delta(\mathcal{S}), e_{v} \notin \mathcal{S}} \theta_{\mathcal{S}, k} \leq M \quad \forall e \in \mathcal{E} \\
\alpha_{k}, \beta_{k e}, \theta_{\mathcal{S}, k} \geq 0
\end{gathered}
$$

Proof: We first assume that all access probabilities ( $p_{k}$ 's) are equal to $p$. Consider the last stage of ECHO in which no new cache locations are opened and the algorithm terminates. At this point, for any node $k, \alpha_{k}=\sum_{e \in \mathcal{E}} p c_{k e} x_{k e}$. Note that $\alpha_{k}=0$ at the end of ECHO for all nodes lying on open edges. For all $k$ with $\alpha_{k}=0$, set $\alpha_{k}=M$. As a result, we have

$$
\begin{aligned}
\sum_{k \in V} \sum_{e \in E} p c_{k e} x_{k e} & \leq \sum_{k \in \mathcal{V}} \alpha_{k} \\
M \sum_{e \in E} z_{e} & \leq \sum_{k \in \mathcal{V}} \alpha_{k} .
\end{aligned}
$$

The inequality in (16) follows from the fact that each node $k$, which lies on an open edge, has $\alpha_{k}=M$.

Set all other dual variables, $\beta$ and $\theta$, to zero. For an edge $e$, define $\mathcal{S}_{e}=\mathcal{E} \backslash\{e\}$. For a node $k$, define $\theta_{\mathcal{S}_{e}, k}$ as follows.

$$
\theta_{\mathcal{S}_{e}, k}= \begin{cases}M & k \in e \\ 0 & \text { otherwise. }\end{cases}
$$

Consider an edge $e \in \mathcal{E}$ and a node $k$ such that $k \notin e$. Suppose $k \in l$. Clearly, at least one such $l$ exists. Then,

$$
\sum_{\mathcal{S} \subseteq \mathcal{E}, e \in \mathcal{S}, e_{v} \notin \mathcal{S}} \theta_{\mathcal{S}, k} \geq \theta_{\mathcal{S}_{l}, k}=M
$$

The last equality follows from (17). Consider now a node $k \in e$. If there exists an edge $l \neq e$ such that $k \in l$, then $\sum_{\mathcal{S} \subset \mathcal{E}, e \in \mathcal{S}, e_{v} \notin \mathcal{S}} \theta_{\mathcal{S}, k} \geq M$. This follows from the same logic as in (18). For a node $k$, which only lies on edge $e$ and no other edge, define $\beta_{k e}=M$. Since, $\alpha_{k} \leq M, \forall k \in \mathcal{V}$ (otherwise the algorithm would not have terminated), the first set of dual feasibility constraints in (12) is satisfied for the assigned values of $\beta$ and $\theta$.

Consider the second set of feasibility constraints in (13). For any edge $e$, we have

$$
\sum_{k \in \mathcal{V}} \beta_{k e} \leq M
$$

This follows directly from the values assigned to the dual variables. For any node $k, \sum_{\mathcal{S} \subseteq \mathcal{E}: e \in \delta(\mathcal{S}), e_{v} \notin \mathcal{S}} \theta_{\mathcal{S}, k}=\theta_{\mathcal{S}_{e}, k}$. Therefore,

$$
\sum_{k \in \mathcal{V} \subseteq} \sum_{\mathcal{S} \subseteq \mathcal{E}: e \in \delta(\mathcal{S}), e_{v} \notin \mathcal{S}} \theta_{\mathcal{S}, k}=\theta_{\mathcal{S}_{e}, r_{e}}+\theta_{\mathcal{S}_{e}, t_{e}}=2 M
$$

Adding (19) and (20), we have

$$
\sum_{k \in \mathcal{V}} \beta_{k e}+\sum_{k \in \mathcal{V}} \sum_{\mathcal{S} \subseteq \mathcal{E}: e \in \delta(\mathcal{S}), e_{v} \notin \mathcal{S}} \theta_{\mathcal{S}, k} \leq 3 M
$$

From above, it is clear that we can obtain a dual feasible solution by dividing all dual variables $(\alpha, \beta, \theta)$ by 3 . Denote these dual feasible variables by $(\hat{\alpha}, \hat{\beta}, \hat{\theta})$. From (15) and (16), we have

$$
\begin{aligned}
\sum_{k \in V} \sum_{e \in E} p c_{k e} x_{k e} & \leq \sum_{k \in \mathcal{V}} \alpha_{k}=3 \times \sum_{k \in \mathcal{V}} \hat{\alpha_{k}} \\
M \sum_{e \in E} z_{e} & \leq \sum_{k \in \mathcal{V}} \alpha_{k}=3 \times \sum_{k \in \mathcal{V}} \hat{\alpha_{k}} .
\end{aligned}
$$

Adding (22) and (23), we have

$$
\sum_{k \in \mathcal{V}} \sum_{e \in \mathcal{E}} p c_{k e} x_{k e}+M \sum_{e \in \mathcal{E}} z_{e} \leq 6 \times \sum_{k \in \mathcal{V}} \hat{\alpha}_{k}
$$


By the theory of duality [20] and (24), we have

$$
\begin{aligned}
\sum_{k \in \mathcal{V}} \hat{\alpha_{k}} \leq O P T & \leq \sum_{k \in \mathcal{V}} \sum_{e \in \mathcal{E}} p c_{k e} x_{k e}+M \sum_{e \in \mathcal{E}} z_{e} \\
& \leq 6 \times O P T .
\end{aligned}
$$

The proof can be extended to the general case when access probabilities are not uniform, by arguments similar to [6].

\section{REFERENCES}

[1] R. Friedman, "Caching web services in mobile ad-hoc networks: opportunities and challenges," in Proc. ACM Int. Workshop on Principles of Mobile Computing (POMC'02), Toulouse, France, Oct. 2002, pp. 90-96.

[2] J. Wang, "A survey of web caching schemes for the Internet," ACM SIGCOMM Comput. Commun. Rev., vol. 29, no. 5, pp. 35-46, Oct. 1999.

[3] B. D. Davison, "A web caching primer," IEEE Internet Comput., vol. 4, no. 4, pp. 38-45, Jul.-Aug. 2001.

[4] D. Kotz and K. Essien, "Analysis of a campus-wide wireless network," in Proc. ACM MOBICOM, Atlanta, GA, Sep. 2002, pp. 107-118.

[5] P. Mirchandani and R. Francis, Discrete Location Theory. New York: Wiley, 1990

[6] C. Swamy and A. Kumar, "Primal-dual algorithms for connected facility location problems," in Proc. 5th Int. Workshop on Approximation Algorithms for Combinatorial Optimization (APPROX'02), Rome, Italy, Sep. 2002, pp. 245-269.

[7] A. Gupta, A. Kumar, and T. Roughgarden, "Simpler and better approximation algorithms for network design," in Proc. 35th Annu. ACM Symp. Theory of Computing (STOC'03), San Diego, CA, 2003, pp. 365-372.

[8] B. Li, M. J. Golin, G. F. Ialiano, and X. Deng, "On the optimal placement of web proxies in the Internet," in Proc. IEEE INFOCOM, New York, Mar. 1999, pp. 1282-1290.

[9] I. Cidon, S. Kutten, and R. Soffer, "Optimal allocation of electronic content," in Proc. IEEE INFOCOM, Anchorage, AK, Apr. 2001, pp. $1773-1780$.

[10] J. Xu, B. Li, and D. L. Lee, "Placement problems for transparent data replication proxy services," IEEE J. Sel. Areas Commun., vol. 20, no. 7, pp. 1383-98, Sep. 2002.

[11] L. Qiu, V. N. Padmanabhan, and G. M. Voelker, "On the placement of web server replicas," in Proc. IEEE INFOCOM, Anchorage, AK, Apr. 2001, pp. $1587-1596$.

[12] Z. Xiang, Z. Zhong, and Y. Zhong, "A cache cooperation management for wireless multimedia streaming," in Proc. IEEE Int. Conf. Info-Tech and Info-Net (ICII'01), Beijing, China, Oct.-Nov. 2001, pp. 328-333.

[13] F. Sailhan and V. Issarny, "Energy-aware web caching for mobile terminals," in Proc. 22nd Int. Conf. Distributed Computing Systems (ICDCS'02), Vienna, Austria, Jul. 2002, pp. 820-825.

[14] Y. Huang, P. Sistla, and O. Wolfson, "Data replication for mobile computers," in Proc. ACM Int. Conf. Management of Data (SIGMOD'94), Minneapolis, MN, Jun. 1994, pp. 13-24.

[15] Bluetooth Core Specification, [Online]. Available: http://www.bluetooth.com/Bluetooth/Learn/Technology/Specifications/

[16] Local and Metropolitan Area Networks: Wireless LAN, ANSI/IEEE Standard 802.11, 1999.

[17] M. Gerla, R. Bagrodia, L. Zhang, K. Tang, and L. Wang, "TCP over wireless multi-hop protocols: simulation and experiments," in Proc. IEEE Int. Conf. Communications, Vancouver, BC, Canada, Jun. 1999, pp. 1089-1094.

[18] P. Winter, "Steiner problem in networks: A survey," ACM Networks, vol. 17, no. 2, pp. 129-167, 1987.

[19] C. E. Perkins, E. M. Royer, and S. Das, "Ad Hoc on Demand Distance Vector (AODV) Routing," IETF RFC 356100, Jul. 2003 [Online]. Available: http://moment.cs.ucsb.edu/AODV/aodv.html\#Publications

[20] V. V. Vazirani, Approximation Algorithms. New York: Springer, 2001.

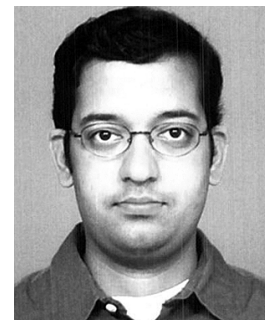

Pavan Nuggehalli (M'98) received the B.S. degree in electrical engineering from the Regional Engineering College at Kurukshetra, India, in 1996, the M.Sc. degree from the Center for Electronics Design and Technology, Indian Institute of Science, Bangalore, in 1998, and the Ph.D. degree in electrical and computer engineering from the University of California at San Diego in 2003.

$\mathrm{He}$ is currently an Assistant Professor at the Center for Electronics Design and Technology, Indian Institute of Science, Bangalore. His research interests are in modeling, analysis, and design of wireless networks.

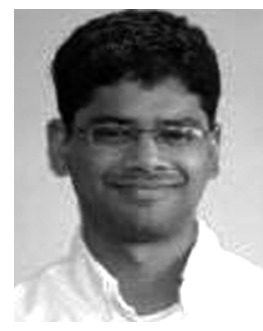

Vikram Srinivasan (M'98) received the B.S. degree in physics from the University of Chennai, India, in 1994, the M.E. degree in electrical communications engineering from the Indian Institute of Science, Bangalore, in 1998, and the Ph.D. degree in electrical and computer engineering from the University of California at San Diego in 2003.

$\mathrm{He}$ is currently an Assistant Professor in the Electrical and Computer Engineering Department, National University of Singapore. His research interests are broadly in the area of wireless networks.

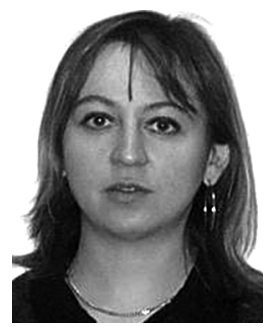

Carla-Fabiana Chiasserini (M'90) graduated with a degree in electrical engineering (summa cum laude) from the University of Florence, Italy, in February 1996, and received the Ph.D. degree in electrical engineering from the Politecnico di Torino in 1999.

Between July and December 1995, she was a Visiting Researcher at the Electrical and Computer Engineering Department, University of Massachusetts, Amherst, where her research focused on security services in wireless LANs. Between May and October 1996, she was a Researcher supported by CSELT (Italian Public Telephone Research Company), developing a handover protocol for wireless ATM networks and computer tools for simulation and performance evaluation. Froom September 1998 to December 1999, she was with the HYPERLINK Center for Wireless Communications at the University of California at San Diego as a Visiting Researcher. Currently, she is an Assistant Professor at the Department of Electronics, Politecnico di Torino. She also collaborates with the San Diego Division of the California Institute of Telecommunications and Information Technologies.

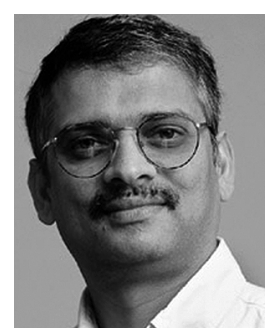

Ramesh R. Rao (SM'90) completed undergraduate work at the Regional Engineering College, University of Madras, Tiruchirapalli, India, and received the B.E. (Honors) degree in electronics and communications in 1980. He received the the M.S. and Ph.D. degrees from the University of Maryland, College Park.

$\mathrm{He}$ is currently a Professor at Irwin and Joan Jacobs School of Engineering, Department of Electrical and Computer Engineering, University of California at San Diego (UCSD), where he has been a member of the faculty since 1984. He is the former Director of UCSD's Center for Wireless Communications (CWC), and currently serves as the Qualcomm Endowed Chair in Telecommunications and Information Technologies, and as the Director of the San Diego Division of the California Institute of Telecommunications and Information Technology (Cal-(IT)2), where he leads several interdisciplinary and collaborative projects. His research interests include architectures, protocols, and performance analysis of computer and communication networks, and he has published extensively on these topics. Since 1984, he has authored over 100 technical papers, contributed book chapters, conducted a number of short courses, and delivered invited talks and plenary lectures. He is currently supervising both masters and doctoral students. 